\title{
Ehrlichia risticii sp. nov.: Etiological Agent of Equine Monocytic Ehrlichiosis (Synonym, Potomac Horse Fever)
}

\author{
CYNTHIA J. HOLLAND, ${ }^{1 *}$ EMILIO WEISS, ${ }^{2}$ WILLY BURGDORFER,${ }^{3}$ ADEYINKA I. COLE, ${ }^{1}$ AND IBULAIMU \\ KAKOMA $^{1}$ \\ Department of Veterinary Pathobiology, College of Veterinary Medicine, University of Illinois, Urbana, Illinois 61801 ${ }^{1}$; \\ Naval Medical Research Institute, Bethesda, Maryland 2081 $4^{2}$; and Rocky Mountain Research Laboratory, Hamilton,
} Montana $59840^{3}$

\begin{abstract}
The etiological agent of equine monocytic ehrlichiosis (synonym, Potomac horse fever) was isolated and propagated in vitro by using techniques similar to those established for some members of the genus Ehrlichia. The organisms, which are contained within a cytoplasmic vacuole, are intermediate in size between Ehrlichia canis and Ehrlichia sennetsu, ranging from 0.4 to $0.75 \mu \mathrm{m}$ wide and from 0.5 to $1.5 \mu \mathrm{m}$ long. The agent is antigenically related to $E$. sennetsu and $E$. canis but also possesses unique antigenic determinants, thereby distinguishing it as a new species of the genus Ehrlichia. The name Ehrlichia risticii is proposed for this new agent. The type strain is strain HRC-IL (= ATCC VR-986).
\end{abstract}

Equine monocytic ehrlichiosis (EME; synonym, Potomac horse fever) is a relatively recently recognized disease of horses. Since the first major outbreak in 1979 within a narrow zone adjacent to the Potomac River in Montgomery County, Md., the geographical distribution and incidence of this disease have increased, with confirmed cases reported in other regions of Maryland, as well as in Virginia, Pennsylvania, New Jersey, and, most recently, Idaho (3; J. E. Palmer, personal communication). Numerous clinically diagnosed but serologically unconfirmed cases have also been reported in many other states and abroad (3).

Clinical symptoms associated with EME vary markedly and may include any or all of the following: fever, depression, anorexia, leukopenia, distal edema of the limbs, laminitis, colic, and mild to severe diarrhea. The mortality rate is more than $30 \%$ in confirmed cases. Preliminary experimental studies have determined that horses which have recovered from the disease are refractory to subsequent infection for an undetermined length of time, although relapse of the disease has been observed (J. E. Palmer, Am. Assoc. Equine Pract. Newsl., p. 63, 1983).

Previous studies revealed that EME is infectious and is transmissible to horses by intravenous inoculation of whole blood obtained from horses during the acute stage of the disease (A. L. Jenny, Am. Assoc. Equine Pract. Newsl., p. 64-65, 1983). In an effort to identify the etiological agent, blood was passaged from infected to susceptible horses on a continuous basis (A. L. Jenny, personal communication). Microscopic examination of infected blood and tissue specimens obtained during the acute phase of the disease, along with numerous bacterial and viral isolation attempts and serological studies, failed to identify a possible causative agent (1, 3, 6; Jenny, Am. Assoc. Equine Pract. Newsl., p. 64-65, 1983).

The possibility of a rickettsial etiology of the disease was suspected after the microscopic observation of a rickettsialike organism in the leukocytes of a horse affected with EME (J. Sessions, personal communication). Sera from both naturally and experimentally infected horses were sent to the laboratory of M. Ristic at the University of Illinois College of Veterinary Medicine to test for antibodies to three agents belonging to the genus Ehrlichia (in particular, Ehrlichia

\footnotetext{
* Corresponding author.
}

equi, the causative agent of equine ehrlichiosis) (11). All of the serum samples were negative for antibodies to $E$. equi (3). However, some reacted at low titers with Ehrlichia canis, the causative agent of canine ehrlichiosis. All serum samples obtained from convalescent horses reacted moderately well against Ehrlichia sennetsu, the agent responsible for human sennetsu rickettsiosis, a disease which is well documented in Japan and may occur in other regions of Southeast Asia $(3,11)$. By contrast, none of the serum samples obtained from horses before experimental infection and from other clinically normal horses which were not known to have experienced the disease reacted with any of the antigens described above (3). Based upon these serological findings, attempts were undertaken at the University of Illinois to isolate the causative agent from the blood of an experimentally infected horse. By using techniques previously developed in the laboratory of $M$. Ristic for the isolation and in vitro cultivation of $E$. canis and $E$. sennetsu $(2,4,8)$, an organism resembling members of the genus Ehrlichia was isolated in monocyte cell cultures obtained from the blood of this animal (C. J. Holland and M. Ristic, Abstr. Conf. Res. Workers Anim. Dis. 1984, p. 34). Inoculation of the cultured organism into a susceptible pony induced a disease which was clinically and pathologically consistent with EME syndrome (3, 5; Jenny, unpublished data). The organism was reisolated in monocyte cultures prepared from the blood of this animal, thereby fulfilling Koch's postulates (3; Holland and Ristic, Abstr. Conf. Res. Workers Anim. Dis. 1984). Almost simultaneously, Rikihisa et al. (10), using electron microscopy, observed intracytoplasmic microorganisms resembling Ehrlichia within macrophage-like cells in the connective tissue of the large colon of an experimentally infected horse. Rikihisa and Perry (9) also reported preliminary observations on the growth of the agent in human histiocytes. More recently, the agent was isolated from an infected horse by using an established mouse macrophage cell line $\left(\mathrm{P} 388 \mathrm{D}_{1}\right)$ by one of us $(\mathrm{E} . \mathrm{W}$., unpublished data) and by S. K. Dutta of the University of Maryland (personal communication). The organisms isolated by these investigators are morphologically indistinguishable from the original blood isolate (3).

Based upon morphological and serological studies between the isolate and established members of the genus Ehrlichia and the genus Rickettsia, we confirmed that the 
etiological agent of EME belongs to the genus Ehrlichia (3, 9,11 ) in the order Rickettsiales (12). Cross-adsorption serological studies (Table 1) confirmed that the etiological agent of EME possesses unique antigenic determinants, warranting its classification as a new species of Ehrlichia. For this new species, we propose the name Ehrlichia risticii sp. nov. (ris. ti. ci' i. N.L. gen. n. risticii in honor of Miodrag Ristic for his many valuable contributions to the field of rickettsiae and rickettsial diseases).

Description of the species. Ehrlichia risticii possesses all of the general phenotypic characteristics common to other members of the genus Ehrlichia $(3,5,9,11)$. The organisms are pleomorphic, appearing as round, oval, or elongated sausage-like structures surrounded by a double plasma membrane and a highly rippled cell wall. Their internal components are highly electron dense and are presumed to consist of deoxyribonucleic acid and ribosomes $(3,10)$. The sizes of individual cells are intermediate between the sizes of $E$. canis and $E$. sennets $u$ cells, ranging from 0.4 to $0.75 \mu \mathrm{m}$ wide and from 0.5 to $1.2 \mu \mathrm{m}$ long $(3,10,11)$. The organisms are contained within a cytoplasmic vacuolar membrane and may occur singly or multiply as inclusion bodies $(3,10)$. Division occurs by binary fission (Fig. 1). The organisms are gram negative and stain bluish purple with Wright Giemsa stain. They cannot be propagated in cell-free media. In vitro cultivation has been established in primary equine and canine monocyte cultures (3) and with murine macrophage cell lines $\mathrm{P}_{388 D_{1}}$ and MDH-SP in medium 199 (GIBCO Laboratories) or RPMI 1640 medium (GIBCO; Quality Biological, Inc.) supplemented with $20 \%$ fetal bovine serum. The optimal growth temperature is 37 to $38^{\circ} \mathrm{C}$ under normal atmospheric conditions (3), but the organism may also be cultured in an humidified atmosphere of $5 \% \mathrm{CO}_{2}$ in air. It can be successfully stored at $-100^{\circ} \mathrm{C}$ as a suspension of infected host cells in tissue culture medium containing 8 to $10 \%$ dimethyl sulfoxide. The organism requires L-glutamine for growth and is severely inhibited by low concentrations of antibiotics, such as tetracycline, penicillin, streptomycin, and gentamicin. As has been reported for E. sennetsu (7), mice are susceptible to infection by $E$. risticii when they are infected intraperitoneally with the blood or buffy coat from an acutely infected horse or with cultured organisms (Jenny, personal communication; A. I. Cole and M. Khondowe, unpublished data; G. S. Miranpuri and W. Burgdorfer, unpublished data).

The susceptibility of other mammalian species and the

TABLE 1. Antigenic differentiation between E. risticii and $E$. sennetsu, as demonstrated by the indirect fluorescent antibody test before and after cross-adsorption of antiserum with homologous and heterologous antigen ${ }^{a}$

\begin{tabular}{llcc}
\hline \multirow{2}{*}{ Antisera } & \multirow{2}{*}{$\begin{array}{c}\text { Adsorbing } \\
\text { antigen }\end{array}$} & $\begin{array}{c}\text { Indirect fluorescent antibody titer with } \\
\text { the following antigens: }{ }^{b}\end{array}$ \\
\cline { 3 - 4 } & & E. risticii & E. sennetsu \\
\hline Anti-E. risticii & None & $160,320,640,1,280$ & $20,40,40,80$ \\
Equine & E. sennetsu & $40,160,640,640$ & $\mathrm{~N}, \mathrm{~N}, \mathrm{~N}, \mathrm{~N}$ \\
Four sera & E. risticii & $10,10,20,40$ & $\mathrm{~N}, \mathrm{~N}, \mathrm{~N}, \mathrm{~N}$ \\
Anti-E. sennetsu & None & 40 & 640 \\
Canine & E. sennetsu & $\mathrm{N}$ & 40 \\
One serum & E. risticii & $\mathrm{N}$ & 320 \\
\hline
\end{tabular}

${ }^{a}$ Undiluted sera were adsorbed with predetermined optimal concentrations of antigen at $37^{\circ} \mathrm{C}$ for $30 \mathrm{~min}$ and centrifuged at $10,000 \times \mathrm{g}$ for $20 \mathrm{~min}$. The supernatant sera were diluted in phosphate-buffered saline $(\mathrm{pH}$ 7.2).

${ }_{b}^{b}$ Expressed as the reciprocal dilution of each serum sample examined. $\mathrm{N}$, Negative at a dilution of 1:10.

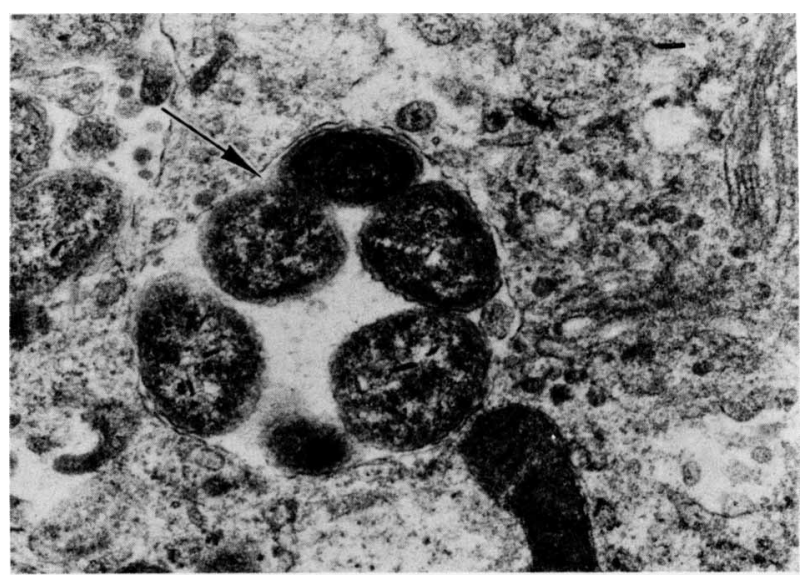

FIG. 1. Ultrathin section of an inclusion body of E. risticii which is contained by a cytoplasmic vacuolar membrane. One of the organisms is at the stage of binary fission (arrow). Bar $=0.1 \mu \mathrm{m}$.

mechanisms by which this organism is transmitted in nature are not known. A blood-sucking arthropod, such as ticks, is strongly suspected in transmission of the disease since experimental infections have only been produced thus far by blood transfusion or intravenous inoculation of cultured organisms.

Within the genus Ehrlichia, E. risticii is more closely related to $E$. sennetsu and $E$. canis, which are also monocytic parasites, than to $E$. equi and, presumably, Ehrlichia phagocytophila, which multiply in granulocytes (11). The guanosine-plus-cytosine contents of the major species of Ehrlichia are currently being investigated.

Description of the type strain. The type strain of $E$. risticii has been deposited with the American Type Culture Collection as strain ATCC VR-986; this strain is the first isolate of E. risticii (= strain HRC-IL) and is described in this paper and elsewhere $(3,5)$.

Serum samples from naturally and experimentally infected horses were received from J. E. Sessions, Glenvilah Veterinary Clinic, Potomac, Md., A. L. Jenny, National Veterinary Services Laboratory, Ames, Iowa, and R. Whitlock, University of Pennsylvania, New Bolton Center, Kennett Square. We thank M. Khondowe, J. Dawson, and I. Abeygunawardena for skillful technical assistance. We gratefully acknowledge D. L. Huxsoll, U.S. Army Medical Research Institute of Infectious Diseases, Fort Detrick, Md., for his part in the initiation of these studies.

This study was supported in part by a grant from the Morris Animal Foundation.

\section{LITERATURE CITED}

1. Ehrich, M., B. D. Perry, H. F. Troutt, R. W. Dellers; and R. A. Magnusson. 1984. Acute diarrhea in horses of the Potomac River area: examination for clostridial toxins. J. Am. Vet. Med. Assoc. 185:433-435.

2. Hoilien, C. A., M. Ristic, D. L. Huxsoll, G. Rapmund, and N. Tachibana. 1982. Rickettsia sennetsu in human blood monocyte cultures: similarities to the growth cycle of Ehrlichia canis. Infect. Immun. 35:314-319.

3. Holland, C. J., M. Ristic, A. I. Cole, P. Johnson, G. Baker, and T. Goetz. 1985. Isolation, experimental transmission, and characterization of causative agent of Potomac horse fever. Science 227:522-524.

4. Holland, C. J., M. Ristic, D. L. Huxsoll, A. I. Cole, and G. Rapmund. 1985. Adaptation of Ehrlichia sennetsu to canine blood monocytes: preliminary structural and serological studies with cell culture-derived Ehrlichia sennetsu. Infect. Immun. 
48:366-371.

5. Holland, C. J., M. Ristic, P. Johnson, G. Baker, and T. Goetz. 1984. Causative agent of Potomac horse fever. Vet. Rec. 115:554-555.

6. Huang, J. C. M., S. L. Wright, and W. D. Shipley. 1983. Isolation of a corona virus-like agent from horses suffering from acute equine diarrhoea. Vet. Rec. 113:262-263.

7. Misao, T. Y., and Y. Kobayashi. 1955. Studies on infectious mononucleosis (glandular fever). Isolation of etiologic agent from blood, bone marrow and lymph node of patients with infectious mononucleosis by using mice. Kyushu J. Med. Sci. 6:145-152.

8. Nyindo, M. B. A., M. Ristic, D. L. Huxsoll, and A. R. Smith.
1971. Tropical canine pancytopenia. In vitro cultivation of the causative agent-Ehrlichia canis. Am. J. Vet. Res. 32:1651-1658.

9. Rikihisa, Y., and B. D. Perry. 1984. Causative agent of Potomac horse fever. Vet. Rec. 115:554.

10. Rikihisa, Y., B. D. Perry, and D. Cordes. 1984. Rickettsial link with acute equine diarrhoea. Vet. Rec. 115:390.

11. Ristic, M., and D. L. Huxsoll. 1984. Genus IV. Ehrlichia, p. 704-709. In N. R. Krieg (ed.), Bergey's manual of systematic bacteriology, vol. 1. The Williams \& Wilkins Co., Baltimore.

12. Weiss, E., and J. M. Moulder. 1984. Order I. Rickettsiales, p. 687-704. In N. R. Krieg (ed.), Bergey's manual of systematic bacteriology, vol. 1. The Williams \& Wilkins Co., Baltimore. 